\title{
Searching for DFT-based methods that include dispersion interactions to calculate the physisorption of $\mathrm{H}_{2}$ on benzene and graphene
}

\author{
I. Cabria, M. J. López, and J. A. Alonso
}

Citation: The Journal of Chemical Physics 146, 214104 (2017); doi: 10.1063/1.4984106

View online: http://dx.doi.org/10.1063/1.4984106

View Table of Contents: http://aip.scitation.org/toc/jcp/146/21

Published by the American Institute of Physics

\section{Articles you may be interested in}

Communication: Density functional theory embedding with the orthogonality constrained basis set expansion procedure

The Journal of Chemical Physics 146, 211101 (2017); 10.1063/1.4984777

Long-range interactions from the many-pair expansion: A different avenue to dispersion in DFT

The Journal of Chemical Physics 146, 024111 (2017); 10.1063/1.4973728

Perspective: Found in translation: Quantum chemical tools for grasping non-covalent interactions

The Journal of Chemical Physics 146, 120901 (2017); 10.1063/1.4978951

Low scaling random-phase approximation electron correlation method including exchange interactions using localised orbitals

The Journal of Chemical Physics 146, 174110 (2017); 10.1063/1.4981817

On the physical interpretation of the nuclear molecular orbital energy

The Journal of Chemical Physics 146, 214103 (2017); 10.1063/1.4984098

Announcement: Top reviewers for The Journal of Chemical Physics 2016

The Journal of Chemical Physics 146, 100201 (2017); 10.1063/1.4978399






\title{
Searching for DFT-based methods that include dispersion interactions to calculate the physisorption of $\mathrm{H}_{2}$ on benzene and graphene
}

\author{
I. Cabria, M. J. López, and J. A. Alonso \\ Departamento de Física Teórica, Atómica y Óptica, Universidad de Valladolid, 47011 Valladolid, Spain
}

(Received 23 March 2017; accepted 12 May 2017; published online 1 June 2017)

\begin{abstract}
Simulations of the hydrogen storage capacities of nanoporous carbons require an accurate treatment of the interaction of the hydrogen molecule with the graphite-like surfaces of the carbon pores, which is dominated by the dispersion forces. These interactions are described accurately by high level quantum chemistry methods, like the Coupled Cluster method with single and double excitations and a non-iterative correction for triple excitations $(\operatorname{CCSD}(\mathrm{T}))$, but those methods are computationally very expensive for large systems and for massive simulations. Density functional theory (DFT)-based methods that include dispersion interactions at different levels of complexity are less accurate, but computationally less expensive. In order to find DFT-methods that include dispersion interactions to calculate the physisorption of $\mathrm{H}_{2}$ on benzene and graphene, with a reasonable compromise between accuracy and computational cost, $\operatorname{CCSD}(\mathrm{T})$, Møller-Plesset second-order perturbation theory method, and several DFT-methods have been used to calculate the interaction energy curves of $\mathrm{H}_{2}$ on benzene and graphene. DFT calculations are compared with $\operatorname{CCSD}(\mathrm{T})$ calculations, in the case of $\mathrm{H}_{2}$ on benzene, and with experimental data, in the case of $\mathrm{H}_{2}$ on graphene. Among the DFT methods studied, the B97D, RVV10, and PBE+DCACP methods yield interaction energy curves of $\mathrm{H}_{2}$-benzene in remarkable agreement with the interaction energy curve obtained with the CCSD(T) method. With regards to graphene, the rev-vdW-DF2, PBE-XDM, PBE-D2, and RVV10 methods yield adsorption energies of the lowest level of $\mathrm{H}_{2}$ on graphene, very close to the experimental data. Published by AIP Publishing. [http://dx.doi.org/10.1063/1.4984106]
\end{abstract}

\section{INTRODUCTION}

Hydrogen is a candidate to replace gasoline in the cars of the future. Hydrogen can be stored in a solid material through different mechanisms: physisorption, chemisorption, and chemical reactions. Among the solid materials investigated, nanoporous carbons attract much attention because these are light materials and have large porosity, and consequently high specific surface areas available for hydrogen adsorption. ${ }^{1-13}$ Nanoporous carbons store hydrogen through physisorption on the walls of the pores. Computer simulations have been performed to interpret the experimental results on hydrogen storage on nanoporous carbons and to make predictions for new carbon-based materials. ${ }^{14-23}$ The theoretical calculations of the hydrogen storage capacities of these materials require an accurate description of the interaction energy curves of a $\mathrm{H}_{2}$ molecule physisorbed on the carbon surfaces. The hydrogen storage capacity of nanoporous carbons depends not only on the region around the minimum of the interaction energy curve, but also on the shape of the interaction energy curve in the region far from the carbon surface, the tail region, especially in the case of wide pores. ${ }^{19,21,22}$

There are two sources of information on the interaction of $\mathrm{H}_{2}$ with carbon-based surfaces: experiments and theoretical calculations. However, the reported experimental and theoretical results vary greatly. The measured adsorption energies of $\mathrm{H}_{2}$ on carbon surfaces vary between 0.035 and $0.110 \mathrm{eV}$ per $\mathrm{H}_{2}$ molecule, because the structure of the carbon surfaces is different. The results from theoretical calculations (adsorption energies, storage capacities, etc.) also vary greatly, depending on the model used to simulate the carbon-based surface and on the theoretical method. Hence, to clarify matters, one should study very specific carbon-based surface models using accurate theoretical methods.

The interaction of $\mathrm{H}_{2}$ with a graphite-like carbon surface is a noncovalent interaction. Long range dispersion forces provide an important contribution to the interaction of $\mathrm{H}_{2}$ with graphitic surfaces. The dispersion forces are weak, and they are difficult to include in the theoretical calculations. High-level $a b$ initio methods such as the Coupled Cluster method with single and double excitations and a non-iterative correction for triple excitations $(\operatorname{CSSD}(\mathrm{T}))^{24}$ give a good account of the dispersion interactions. Møller-Plesset second-order perturbation theory method (MP2) ${ }^{25}$ does not reproduce well screening effects and sometimes is affected by important errors also in small systems, like the benzene dimer. On the other hand, the local density approximation (LDA) and the generalized gradient approximation (GGA) versions of the density functional theory (DFT) do not include dispersion interactions. Nowadays, the best method to treat noncovalent interactions is $\operatorname{CCSD}(\mathrm{T}){ }^{26-28}$ Both CCSD (T) and MP2 methods are expected to yield accurate interaction energy curves for $\mathrm{H}_{2}$ on a graphitic carbon surface, but these two methods are computationally expensive and are therefore restricted to small systems. The computational cost of the CCSD(T), MP2, and DFT methods scales as $\mathrm{O}\left(\mathrm{N}^{7}\right), \mathrm{O}\left(\mathrm{N}^{5}\right)$, and $\mathrm{O}\left(\mathrm{N}^{3}\right)$, respectively, where $N$ is proportional to the system size. Then, it would be desirable to 
find DFT-based methods that include dispersion corrections and reproduce accurately the $\operatorname{CCSD}(\mathrm{T})$ interaction energy curve of $\mathrm{H}_{2}$ on a carbon surface, with a compromise between accuracy and computational cost.

In the last fifteen years, DFT-based methods including dispersion interactions have been developed and tested in many dispersion-dominated systems. Klimeš and Michaelides ${ }^{29}$ have reviewed the different DFT-based methods that include dispersion. One class of those DFT-based methods uses energy functionals that do not depend on external input parameters, and the dispersion interactions are obtained directly from the electron density. Those functionals are termed non-local correlation functionals because non-local correlations are added to local or semi-local correlation functionals. Among the methods based on non-local DFT functionals are the van der Waals Density Functional (vdW-DF) schemes ${ }^{30-38}$ and the VV10 $0^{39}$ and RVV10 methods. ${ }^{40}$

A second class of DFT-based methods uses functionals which include the dispersion interactions empirically or semiempirically at different levels of complexity. Among those methods are the DFT-D schemes, ${ }^{41}$ the DFT+DCACP method, ${ }^{42-46}$ which is based on dispersion corrected atom centered pseudopotentials (DCACPs), the Tkatchenko-Scheffler (TS) method, ${ }^{47}$ the exchange-hole dipole moment (XDM) method, ${ }^{48,49}$ and the vdW/DFT-WF method, ${ }^{50-54}$ which is based on maximally localized Wannier functions. DFT-D stands for Density Functional Theory with empirical Dispersion corrections. In the DFT-D scheme, an energy term is added, taking into account the dispersion interactions. That energy term includes some constants whose values are obtained empirically and remain constant throughout the calculation.

Dispersion can also be modelled by using effective atomcentered nonlocal pseudopotentials, such as the dispersion corrected atom centered pseudopotentials, DCACPs, developed by Rothlisberger and co-workers. ${ }^{42-46}$ The parameters of these pseudopotentials were obtained by fitting the values of the properties of weakly bonded dimers of $\mathrm{H}_{2}$, benzene, $\mathrm{N}_{2}, \mathrm{CO}_{2}, \mathrm{He}, \mathrm{Ne}, \mathrm{Ar}$, and $\mathrm{Kr}^{45,46}$ to the results of highlevel $a b$ initio calculations. The calculations are done with the unmodified density functional plus the corresponding DCACPs, and this is called the DFT+DCACP method. In the present work, we have performed $\mathrm{PBE}+\mathrm{DCACP}$ calculations.

Comparisons of the binding energies, equilibrium geometries, and interaction energy curves of systems bonded by dispersion interactions, obtained with $\operatorname{CCSD}(\mathrm{T}), \mathrm{MP} 2$, DFT, DFT-D, and vdW-DF methods, have been published. ${ }^{19,26,30,36,54-62}$ The interaction energy curve between $\mathrm{H}_{2}$ and a carbon surface is a basic ingredient in the models developed to predict the hydrogen storage capacities of porous carbons. ${ }^{17,18,21,63}$ Some previous publications compared the interaction energy curves of $\mathrm{H}_{2}$ on benzene, graphene, and nanotubes. Mpourmpakis and Froudakis ${ }^{19}$ have compared the interaction energy curves of $\mathrm{H}_{2}$ with $(6,6),(9,9)$, and $(11,11)$ carbon nanotubes obtained with MP2 and DFT methods, modeling the nanotubes as $\mathrm{C}_{24} \mathrm{H}_{12}$ clusters with the curvature of the respective nanotubes. The DFT methods tested included LDA, GGA, and hybrid functionals, but dispersion interactions were not included. They also performed CCSD calculations, but only at two separation distances. Costanzo et al. ${ }^{52}$ compared the interaction energy curves of $\mathrm{H}_{2}$ on graphene obtained with a DFT-D, a vdW-DF method, and the DFT/vdW-WF method.

There are studies and comparisons of $\mathrm{H}_{2}$ physisorbed on benzene and graphene in the scientific literature, but as far as we know, those studies involve only some functionals that include dispersion interactions. There is a lack of a comparison of the interaction energy curves of $\mathrm{H}_{2}$ on benzene and graphene obtained with $\operatorname{CCSD}(\mathrm{T})$ and with the main DFT-based methods that include dispersion interactions. The purpose of the present work is to make that comparison for the main and most popular DFT-based methods that include dispersion interactions, in order to evaluate their performance to describe the physisorption of molecular hydrogen on benzene and graphene.

Benzene is the simplest carbon-based surface, and graphene is one of the largest carbon-based surfaces. Benzene is a small molecule, and therefore it is feasible to make accurate $\operatorname{CCSD}(\mathrm{T})$ calculations of the interaction of $\mathrm{H}_{2}$ on benzene. The $\mathrm{H}_{2}$-benzene interaction energy curves obtained with DFT-based methods will be compared with the CCSD(T) interaction energy curve, the best theoretical curve. On the other hand, the DFT calculations of the $\mathrm{H}_{2}$-graphene system will be compared with experimental data, in particular, with the experimental adsorption energy of the lowest level of $\mathrm{H}_{2}$ physisorbed on graphene. ${ }^{64}$

In the present work, the results of $\operatorname{CCSD}(\mathrm{T}),{ }^{24} \mathrm{MP} 2,{ }^{25}$ PBE, ${ }^{65,66} \mathrm{VWN},{ }^{67}$ PW91, ${ }^{68}$ PBE+DCACP, ${ }^{42-45}$ B97D, ${ }^{41,69}$ PBE-D2, ${ }^{41}$ PBE-TS, ${ }^{47}$ PBE-XDM, ${ }^{48,49}$ vdW-DF, ${ }^{30}$ optB88vdW ${ }^{32}$ optB86b-vdW, ${ }^{33}$ vdW-DF-cx, ${ }^{34}$ vdW-DF-C09, ${ }^{35}$ vdW-DF $2,{ }^{36}$ vdW-DF2-C09, ${ }^{37}$ rev-vdW-DF2, ${ }^{38}$ and RVV $10^{40}$ calculations of $\mathrm{H}_{2}$ physisorbed on benzene and the results of PBE, PBE+DCACP, PBE-D2, PBE-TS, PBE-XDM, vdWDF, optB88-vdW, optB86b-vdW, vdW-DF-cx, vdW-DF-C09, vdW-DF2, vdW-DF2-C09, rev-vdW-DF2, and RVV10 calculations of $\mathrm{H}_{2}$ physisorbed on graphene are presented. The calculations have been performed with three computational codes: Gaussian 09, ${ }^{70}$ BigDFT, $^{71-73}$ and Quantum Espresso, QE. ${ }^{74,75}$ In Sec. II, the computational codes and the parameters used are briefly presented. Section III consists of the discussion and comparison of the interaction energy curves of $\mathrm{H}_{2}$ on benzene and graphene, obtained with all the methods. The conclusions are presented in Sec. IV.

\section{COMPUTATIONAL METHODS}

Three codes have been used to carry out the present calculations of $\mathrm{H}_{2}$ on benzene and graphene: Gaussian 09 (G09), BigDFT, and Quantum Espresso (QE). DFT and DFT-based methods that include dispersion interactions are implemented in these codes. We have followed the same procedures with the three codes: For each of the methods used, the geometries of $\mathrm{H}_{2}$, benzene, and graphene were optimized. The geometry optimization was performed until the forces acting on the atoms were less than the threshold values depending on the code. See the specific values in the corresponding subsections. 
After the optimization of the geometries of $\mathrm{H}_{2}$, benzene, and graphene, the calculations of $\mathrm{H}_{2}$ interacting on benzene and graphene were carried out, for each method. In the case of $\mathrm{H}_{2}$ on benzene, the hydrogen molecule was placed on top of the center of the benzene molecule and perpendicularly oriented with respect to the benzene plane (see Fig. 1). This orientation yields the lowest binding energy of $\mathrm{H}_{2}$ on benzene. The individual geometries of benzene or graphene and $\mathrm{H}_{2}$ were kept frozen, with the optimized geometries obtained in the previous step for the corresponding method, and only the distance between the center of mass of $\mathrm{H}_{2}$ and the benzene or graphene plane was changed. That distance was changed from 2 to $7 \AA$, for benzene, and from 2 to $6 \AA$, for graphene, with a step of $0.1 \AA$. In the case of $\mathrm{H}_{2}$ on graphene, the hydrogen molecule was placed on top of three sites and with three different orientations of the $\mathrm{H}_{2}$ axis with respect to the graphene plane.

The approximation of keeping frozen the individual geometries of benzene, graphene, and $\mathrm{H}_{2}$, and changing only the benzene- $\mathrm{H}_{2}$ or graphene- $\mathrm{H}_{2}$ distance, to study the physisorption was checked. Relaxation of all the coordinates of the systems $\mathrm{H}_{2}$ on benzene and $\mathrm{H}_{2}$ on graphene, around the minimum of their physisorption energy curves and for a single method, RVV10, was carried out. The minimum is located at a benzene- $\mathrm{H}_{2}$ distance of $3.1 \AA$, with $\mathrm{H}_{2}$ on top of the center of the benzene molecule and perpendicular to the benzene plane, and at a graphene- $\mathrm{H}_{2}$ distance of $2.96 \AA$, with $\mathrm{H}_{2}$ on top of the center of a hexagon and parallel to the benzene plane, for the RVV10 method. This relaxation yielded a geometry with a total energy of about $10^{-4} \mathrm{eV}$ lower than the binding energy obtained by keeping frozen the individual geometries and relaxing only the benzene- $\mathrm{H}_{2}$ or the graphene- $\mathrm{H}_{2}$ distance. This difference is very small compared to the binding energy of $\mathrm{H}_{2}$ on benzene, $-0.0450 \mathrm{eV}$, and on graphene, $-0.0629 \mathrm{eV}$. Therefore, the approximation of keeping frozen the individual geometries is a valid approximation to study the physisorption, taking into account that the physisorption occurs at $\mathrm{H}_{2}$-surface distances equal or larger than approximately 2.4-2.5 $\AA$. At very short $\mathrm{H}_{2}$-surface distances, the interaction is strongly repulsive and the approximation will not be valid. Chemisorption effects occur at very short distances and this work is devoted only to physisorption effects.

The interaction energy $E(d)$ between benzene or graphene and a $\mathrm{H}_{2}$ molecule placed at a distance $d$ from the benzene or graphene plane is defined in terms of the energies of the interacting system and the separated components as



FIG. 1. Lateral and top views of the $\mathrm{H}_{2}$ molecule on top of the benzene molecule and perpendicular to the benzene-plane.

$$
E(d)=E\left(H_{2} \text { on } \mathrm{A} ; d\right)-E\left(H_{2}\right)-E(\mathrm{~A}),
$$

where $A$ is benzene or graphene. The binding energy $E_{b}$ is the interaction energy at the equilibrium $\mathrm{H}_{2}$-benzene or $\mathrm{H}_{2}$ graphene plane distance $d_{e}$, i.e., $E_{b}=E\left(d_{e}\right)$. A negative value of $E_{b}$ means that the molecule is bonded to the benzene molecule or to graphene.

\section{A. Gaussian 09}

The Gaussian 09 (G09) code $^{70}$ was used to make calculations of $\mathrm{H}_{2}$ on benzene, but not on graphene. Several methods implemented in the G09 package have been used in the present work: $\mathrm{CCSD}(\mathrm{T}),{ }^{24} \mathrm{MP} 2,{ }^{25} \mathrm{PBE},{ }^{65,66} \mathrm{VWN},{ }^{67}$ PW91, ${ }^{68}$ B97D, ${ }^{41,69}$ and PBE-D2. ${ }^{41}$ The VWN method uses the LDA for exchange and correlation effects with the VoskoWilk-Nusair (VWN) parameterization. ${ }^{67} \mathrm{PBE}$ and PW91 use GGA functionals due to Perdew, Burke, and Ernzerhof, ${ }^{65,66}$ and to Perdew and Wang, ${ }^{68}$ respectively. PBE-D2 and B97D include dispersion corrections according to the DFT-D scheme. PBE-D2 stands for the PBE functional and the Grimme's D2 dispersion corrections. ${ }^{41}$ B97D is a functional proposed by Grimme, ${ }^{41}$ based on the B97 functional of Becke ${ }^{69}$ and Grimme's D2 dispersion corrections. It is a reparameterization of the original B97 functional.

This code performs all-electron calculations using Gaussian basis sets to expand the wavefunctions. The basis set used for all the methods is the augmented correlation-consistent basis set, aug-cc-pVTZ, of Dunning et al. ${ }^{76-80}$ Basis sets of the aug-cc-pVXZ family contain diffuse functions which are necessary to account for dispersion interactions. The spin restricted calculations (because $\mathrm{H}_{2}$ and benzene are closedshell systems) were done self-consistently with a total energy convergence tolerance of $2.72 \times 10^{-5} \mathrm{eV}$. The optimizations of the geometries were run until the forces on the atoms were lower than $4.63 \times 10^{-3} \mathrm{eV} / \AA$ and the displacements of atoms were lower than $1.9 \times 10^{-4} \AA$.

Dispersion energies are small. For this reason, the interaction energies have been calculated always using the counterpoise correction method of Boys and Bernardi ${ }^{81}$ in order to correct the basis set superposition error (BSSE). This has been done for all the methods used with the G09 code.

A test of the basis set is provided in Fig. 2, where a comparison of CCSD(T) calculations of $\mathrm{H}_{2}$ interacting with benzene, done with the aug-cc-pVDZ, aug-cc-pVTZ, and aug-cc-pVQZ basis sets, is presented. The counterpoise method was used to calculate the interaction energies in Fig. 2. The aug-ccpVQZ basis set is the largest one. The difference between the aug-cc-pVTZ and aug-cc-pVQZ interaction energies is smaller than $10^{-3} \mathrm{eV} /$ molecule in the region near the minimum, and it becomes negligible at larger $\mathrm{H}_{2}$-benzene distances. The $\operatorname{CCSD}(\mathrm{T})$ interaction energies obtained with the aug-cc-pVQZ basis set are very similar to those obtained using the aug-ccpVTZ basis set (see Fig. 2), but the computational cost was much higher. Therefore, we selected the aug-cc-pVTZ basis set and the counterpoise method to make the G09 calculations with all the methods.

The use of aug-cc-pVTZ basis sets, the correction of the BSSE, and the value of the self-consistent threshold used 


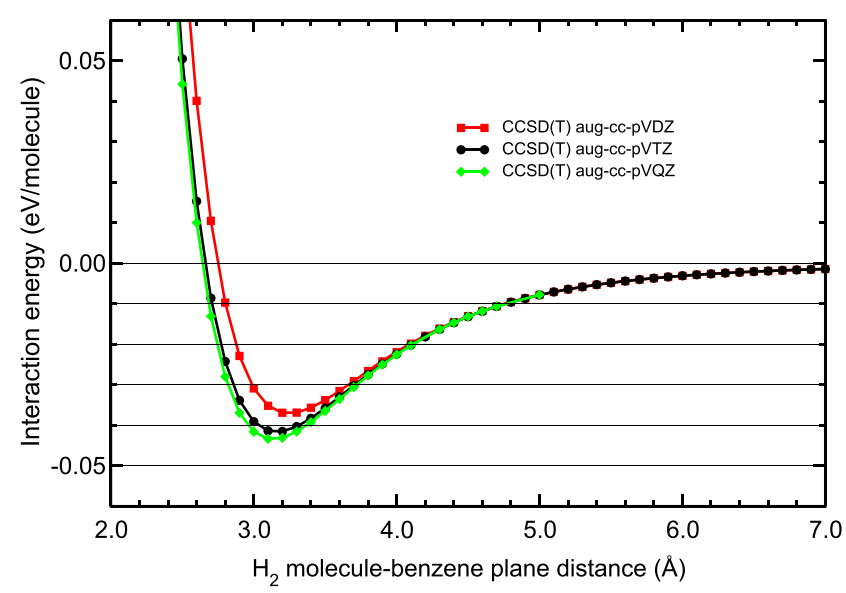

FIG. 2. Interaction energy between $\mathrm{H}_{2}$ and benzene as a function of the $\mathrm{H}_{2}$ benzene distance, obtained by the $\operatorname{CCSD}(\mathrm{T})$ method and the counterpoise method, as implemented in the Gaussian 09 code. Calculations performed with the aug-cc-pVDZ, aug-cc-pVTZ, and aug-cc-pVQZ basis sets are compared.

mean that the present G09 calculations have a high degree of precision.

$\operatorname{CCSD}(\mathrm{T})$ calculations of $\mathrm{H}_{2}$ parallel to the benzene plane, using the aug-cc-pVTZ basis set and the counterpoise method, yielded a less negative binding energy, $-0.0288 \mathrm{eV}$, than the $\operatorname{CCSD}(\mathrm{T})$ calculations of $\mathrm{H}_{2}$ perpendicular to the benzene plane in the same conditions, $-0.0415 \mathrm{eV}$. $\mathrm{H}_{2}$ perpendicular is more stable than $\mathrm{H}_{2}$ parallel, and therefore, we have chosen the $\mathrm{H}_{2}$ perpendicular orientation to make the calculations with all the methods.

\section{B. BigDFT}

The BigDFT code ${ }^{71-73}$ was used to perform PBE+DCACP calculations of $\mathrm{H}_{2}$ on benzene and graphene. This code $\mathrm{e}^{71-73}$ replaces the core electrons by pseudopotentials and expands the electronic wavefunctions in a basis set of wavelets. The code includes only DFT-based methods. The calculations were done self-consistently with a convergence tolerance of 2.72 $\times 10^{-6} \mathrm{eV}$. Spin-restricted calculations have been carried out. The parameters taken to select the basis wavelets were as follows: a grid spacing of $0.15 \mathrm{bohr}$ for benzene and $0.25 \mathrm{bohr}$ for graphene in the three spatial directions and the values of 9.5 and 9.0 for the coarse and fine grid radius multipliers, respectively. These multipliers are the factors multiplying the coarse and fine radius around each atom. These values were chosen after carrying out tests of the convergence of the interaction energy with respect to the values of the parameters. With these values of the parameters of the basis wavelets, the interaction energies $E(d)$ have a precision of $10^{-4} \mathrm{eV}$. DCACPs developed by Rothlisberger and co-workers ${ }^{42-45}$ were used with the PBE functional. These calculations are called PBE+DCACP throughout the paper.

The geometries of the hydrogen molecule, benzene, and graphene were considered optimized when the forces on the atoms were less than $2 \times 10^{-3} \mathrm{eV} / \AA$. Calculations of $\mathrm{H}_{2}$ and benzene were done in free boundary conditions. The unit cell for the graphene periodic calculations was a planar layer of 32 carbon atoms and a lattice parameter of $20 \AA$ in the direction perpendicular to the graphene plane. The lattice parameter of $20 \AA$ was chosen after carrying out tests of the convergence of the interaction energy with respect to the lattice parameter.

\section{Quantum Espresso}

The Quantum Espresso code was used to make calculations of $\mathrm{H}_{2}$ physisorbed on benzene with the functionals PBE$\mathrm{TS},{ }^{47}$ PBE-XDM,${ }^{48,49}$ vdW-DF, ${ }^{30}$ optB88-vdW, ${ }^{32}$ optB86bvdW, ${ }^{33}$ vdW-DF-cx, ${ }^{34}$ vdW-DF-C0 $9,{ }^{35}$ vdW-DF2, ${ }^{36}$ vdWDF2-C09, ${ }^{37}$ rev-vdW-DF2, ${ }^{38}$ and RVV10, ${ }^{40}$ and to make calculations of $\mathrm{H}_{2}$ physisorbed on graphene with the functionals PBE ${ }^{65,66}$ PBE-D2 ${ }^{41}$ PBE-TS, PBE-XDM, vdW-DF, optB88vdW, optB86b-vdW, vdW-DF-cx, vdW-DF-C09, vdW-DF2, vdW-DF2-C09, rev-vdW-DF2, and RVV10. The works of Thonhauser et al. and Román-Pérez and Soler are relevant in the practical implementation of the vdW-DF functionals. ${ }^{82,83}$ This code uses planewaves basis sets. The kinetic energy cutoff for the wavefunctions was set at 120 Ry and the kinetic energy cutoff for charge density and potential was set at $480 \mathrm{Ry}$, after carrying out tests of the convergence of the interaction energy with respect to the cutoffs. With these values of the cutoffs, the precision of the interaction energies $E(d)$ is $10^{-4} \mathrm{eV}$. All the calculations were performed using the Martins-Troullier norm-conserving pseudopotentials, ${ }^{84}$ except the PBE-XDM calculations, which were performed using the Kresse-Joubert Projector Augmented-Wave, PAW, pseudopotentials. ${ }^{85}$ Only the $\Gamma$ point was used in the calculations of both benzene and graphene.

The unit cell for the benzene calculations was a cubic supercell with a lattice parameter of $15 \AA$. The unit cell for the graphene calculations was a hexagonal planar layer of 72

TABLE I. Equilibrium $\mathrm{H}-\mathrm{H}$ distance in $\mathrm{H}_{2}, \mathrm{C}-\mathrm{C}$, and $\mathrm{C}-\mathrm{H}$ distances in benzene and $\mathrm{C}-\mathrm{C}$ distance in graphene, in $\AA$, obtained with different methods.

\begin{tabular}{lcccc}
\hline \hline Method & $d_{\mathrm{H}-\mathrm{H}}$ & $\begin{array}{c}d_{\mathrm{C}-\mathrm{C}} \\
\text { benzene }\end{array}$ & $\begin{array}{c}d_{\mathrm{C}-\mathrm{H}} \\
\text { benzene }\end{array}$ & $\begin{array}{c}d_{\mathrm{C}-\mathrm{C}} \\
\text { graphene }\end{array}$ \\
\hline CCSD(T) & 0.7430 & 1.3980 & 1.0839 & $\ldots$ \\
MP2 & 0.7374 & 1.3942 & 1.0822 & $\ldots$ \\
VWN & 0.7660 & 1.3858 & 1.0937 & $\ldots$ \\
PW91 & 0.7494 & 1.3959 & 1.0890 & $\ldots$ \\
PBE & 0.7509 & 1.3974 & 1.0909 & 1.4196 \\
\hline PBE+DCACP & 0.7505 & 1.3973 & 1.0909 & 1.4284 \\
B97D & 0.7449 & 1.3988 & 1.0873 & $\ldots$ \\
PBE-D2 & 0.7509 & 1.3982 & 1.0910 & 1.4195 \\
PBE-TS & 0.7424 & 1.3917 & 1.0852 & 1.4180 \\
PBE-XDM & 0.7505 & 1.3963 & 1.0905 & 1.4243 \\
\hline vdW-DF & 0.7313 & 1.3909 & 1.0797 & 1.4197 \\
vdW-DF-C09 & 0.7456 & 1.3906 & 1.0884 & 1.4180 \\
vdW-DF-cx & 0.7444 & 1.3908 & 1.0879 & 1.4181 \\
optB86b-vdW & 0.7421 & 1.3906 & 1.0863 & 1.4183 \\
optB88-vdW & 0.7372 & 1.3895 & 1.0825 & 1.4178 \\
\hline vdW-DF2 & 0.7276 & 1.3927 & 1.0772 & 1.4224 \\
rev-vdW-DF2 & 0.7425 & 1.3910 & 1.0864 & 1.4186 \\
vdW-DF2-C09 & 0.7450 & 1.3912 & 1.0882 & 1.4185 \\
RVV10 & 0.7363 & 1.3935 & 1.0825 & 1.4218 \\
\hline Reference & $0.7414^{86}$ & $1.3902^{87}$ & $1.0862^{87}$ & $1.42^{88}$ \\
\hline \hline
\end{tabular}


carbon atoms, with a lattice parameter of $15 \AA$ in the direction perpendicular to the graphene plane. The lattice parameter of $15 \AA$ for benzene and graphene was chosen after carrying out tests of the convergence of the interaction energy with respect to the lattice parameter. The SCF procedure was considered converged if the absolute total energy change was below $1.36 \times 10^{-6} \mathrm{eV}$ for benzene calculations and $1.36 \times$ $10^{-7} \mathrm{eV}$ for graphene calculations. The geometries were considered converged when the forces on the atoms were less than $2.57 \times 10^{-4} \mathrm{eV} / \AA$.

The results of the optimization of the geometries of $\mathrm{H}_{2}$, benzene, and graphene carried out with the $\operatorname{CSSD}(\mathrm{T})$ and MP2 methods and with different functionals are reported in Table I. The theoretical or experimental references for the geometries are at the bottom of that table. The equilibrium $\mathrm{H}-\mathrm{H}$ distance in the $\mathrm{H}_{2}$ molecule is $0.7414 \AA$ according to high precision Hylleraas variational calculations. ${ }^{86}$ Experimental values of the geometry of benzene, $d_{C-C}=1.3902 \pm 0.0002 \AA$ and $d_{C-H}$ $=1.0862 \pm 0.0015 \AA$, were obtained from the work of Plíva et al. ${ }^{87}$ The experimental carbon-carbon distance is $1.42 \AA$ in graphite. ${ }^{88}$

\section{CALCULATIONS OF $\mathrm{H}_{2}$ PHYSISORBED ON BENZENE: MP2 AND DFT-BASED METHODS VS CCSD(T) METHOD}

The interaction energy curves obtained in the calculations are plotted in Figs. 3-5. Some horizontal lines corresponding to $0,-0.01,-0.02,-0.03,-0.04$, and $-0.05 \mathrm{eV}$ have been plotted in those figures, to guide the eye in the comparison of the different curves. The $\mathrm{H}_{2}$-benzene plane equilibrium distances and the binding energies obtained with the different methods are given in Table II.

The $\operatorname{CSSD}(\mathrm{T})$ results (binding energy, equilibrium $\mathrm{H}_{2}-$ plane distance, and interaction energy curve) are the benchmark and the results obtained with the other methods will be compared with the CCSD(T) results. To make these comparisons possible, the $\operatorname{CCSD}(\mathrm{T})$ interaction energy curve is plotted in the three figures of the interaction energy curves: Figures $3-5$. The $\operatorname{CCSD}(\mathrm{T})$ method yields a binding energy $E_{b}=-0.0415 \mathrm{eV}$ and an equilibrium distance $d_{e}=3.2 \AA$.

The binding energies and the equilibrium distances are features of a single point of the interaction energy curve: its minimum. However, to study the physisorption and the hydrogen storage, two regions in the interaction energy curve must be considered and not only the single point of the minimum: The region around the minimum of the curve, approximately between $d_{i}=2.8$ and $4.0 \AA$, and the tail region of the curve, above $d_{i}=4.0 \AA$, where $d_{i}$ is the $\mathrm{H}_{2}$-benzene surface distance.

The interaction energy curves in Figs. 3-5 can be compared visually with the interaction energy curve obtained in $\operatorname{CCSD}(\mathrm{T})$ calculations. That visual comparison is qualitative and in some cases is not accurate. In order to compare quantitatively and more accurately the curves, we have defined the $\mathrm{RMSE}_{m}$, the root-mean-square error, and the RMSPE ${ }_{m}$, the root-mean-square percentage error, of the region around the minimum of the interaction energy curve, obtained with the method F, as
TABLE II. Equilibrium $\mathrm{H}_{2}$-benzene plane distances, $d_{e}$, in $\AA$, and binding energies, $E_{b}$, in $\mathrm{eV}$, for $\mathrm{H}_{2}$ perpendicular to the benzene molecule obtained with different methods. $\mathrm{RMSE}_{m}$ and $\mathrm{RMSE}_{t}$ are in $\mathrm{eV}$, and $\mathrm{RMSPE}_{m}$ and $\mathrm{RMSPE}_{t}$ are in \% (see text for their meaning).

\begin{tabular}{lcccccc}
\hline \hline Method & $d_{e}$ & $E_{b}$ & $\mathrm{RMSE}_{m}$ & $\mathrm{RMSPE}_{m}$ & $\mathrm{RMSE}_{t}$ & $\mathrm{RMSPE}_{t}$ \\
\hline CCSD(T) & 3.2 & -0.0415 & 0.0000 & 0 & 0.0000 & 0 \\
MP2 & 3.1 & -0.0487 & 0.0064 & 21 & 0.0006 & 7 \\
VWN & 2.8 & -0.0996 & 0.0365 & 118 & 0.0015 & 35 \\
PW91 & 3.4 & -0.0290 & 0.0136 & 43 & 0.0012 & 27 \\
PBE & 3.4 & -0.0204 & 0.0212 & 66 & 0.0030 & 37 \\
\hline PBE+DCACP & 3.3 & -0.0413 & 0.0036 & 13 & 0.0011 & 33 \\
B97D & 3.1 & -0.0371 & 0.0035 & 9 & 0.0010 & 12 \\
PBE-D2 & 3.0 & -0.0565 & 0.0132 & 44 & 0.0007 & 8 \\
PBE-TS & 3.2 & -0.0561 & 0.0100 & 28 & 0.0014 & 13 \\
PBE-XDM & 3.2 & -0.0538 & 0.0111 & 34 & 0.0032 & 60 \\
\hline vdW-DF & 3.5 & -0.0450 & 0.0168 & 61 & 0.0073 & 87 \\
vdW-DF-C09 & 3.3 & -0.0379 & 0.0058 & 20 & 0.0055 & 83 \\
vdW-DF-cx & 3.6 & -0.0405 & 0.0130 & 46 & 0.0066 & 82 \\
optB86b-vdW & 3.3 & -0.0413 & 0.0066 & 24 & 0.0049 & 72 \\
optB88-vdW & 3.3 & -0.0390 & 0.0055 & 19 & 0.0029 & 52 \\
\hline vdW-DF2 & 3.3 & -0.0448 & 0.0063 & 22 & 0.0013 & 11 \\
rev-vdW-DF2 & 3.3 & -0.0359 & 0.0073 & 24 & 0.0007 & 7 \\
vdW-DF2-C09 & 3.7 & -0.0168 & 0.0275 & 84 & 0.0013 & 11 \\
RVV10 & 3.1 & -0.0450 & 0.0029 & 9 & 0.0004 & 12 \\
\hline \hline
\end{tabular}

$$
\begin{gathered}
R M S E_{m}=\sqrt{\sum_{i=1}^{N} \frac{\left(E\left(d_{i}\right)_{\mathrm{F}}-E\left(d_{i}\right)_{\mathrm{CCSD}(\mathrm{T})}\right)^{2}}{N}}, \\
R M S P E_{m}=100 \sqrt{\sum_{i=1}^{N} \frac{\left(E\left(d_{i}\right)_{\mathrm{F}}-E\left(d_{i}\right)_{\mathrm{CCSD}(\mathrm{T})}\right)^{2}}{\left(E\left(d_{i}\right)_{\mathrm{CCSD}(\mathrm{T})}\right)^{2} N}},
\end{gathered}
$$

where $d_{i}$ runs from $d_{1}=2.8 \AA$ to $d_{N}=4.0 \AA$, with a step of $0.01 \AA$, and $E\left(d_{i}\right)_{\mathrm{F}}$ and $E\left(d_{i}\right)_{\mathrm{CCSD}(\mathrm{T})}$ are the interaction energies of $\mathrm{H}_{2}$ on benzene, for a $\mathrm{H}_{2}$-benzene surface distance of $d_{i}$, obtained with the methods $\mathrm{F}$ and $\operatorname{CCSD}(\mathrm{T})$, respectively. $\mathrm{RMSE}_{m}$ has the same units as $E\left(d_{i}\right), \mathrm{eV}$, and $\mathrm{RMSPE}_{m}$ is in \%. We have also defined the RMSE ${ }_{t}$ and $\mathrm{RMSPE}_{t}$ quantities as the root-mean-square error and root-mean-square percentage error, respectively, of the tail region of the interaction energy curve. $\mathrm{RMSE}_{t}$ and $\mathrm{RMSPE}_{t}$ are also defined according to Eqs. (2) and (3), respectively, but replacing $m$ by $t$ and $d_{i}$ running from $d_{1}=4.1 \AA$ to $d_{N}=7.0 \AA$, with a step of $0.01 \AA$.

RMSE is a measure of the similarity between the interaction energy curve obtained with a particular method and the $\operatorname{CCSD}(\mathrm{T})$ interaction energy curve. The smaller the value of RMSE, the closer the similarity between the two curves. $\mathrm{RMSE}_{x}$ can be also considered as the average difference between the interaction energies of the $\mathrm{F}$ and $\operatorname{CCSD}(\mathrm{T})$ methods in the region around the minimum $(\mathrm{x}=\mathrm{m})$ or in the tail region $(x=t)$. The calculated values of $\mathrm{RMSE}_{m}, \mathrm{RMSPE}_{m}$, $\mathrm{RMSE}_{t}$, and $\mathrm{RMSPE}_{t}$ are given in Table II.

The methods studied have been separated into three groups. The first group is composed by the MP2 method and by DFT-based methods that do not include the dispersion interactions. In this group are the VWN, PW91, and PBE methods. Figure 3 contains the interaction energy curves of the first 


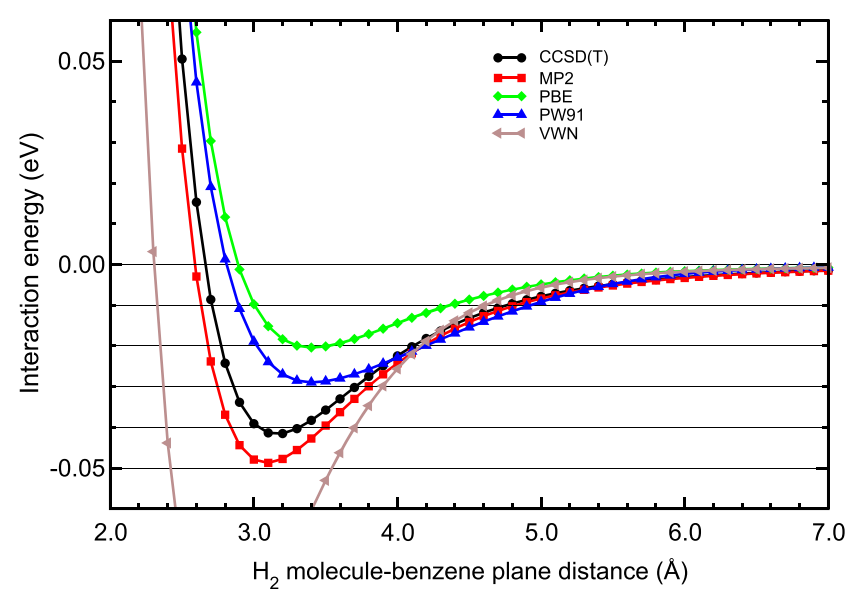

FIG. 3. Interaction energy between $\mathrm{H}_{2}$ and benzene as a function of the $\mathrm{H}_{2}$ benzene plane distance, obtained in CCSD(T), MP2, and DFT (VWN, PW91, and PBE) calculations.

group. The second group is composed by DFT-based methods that include the dispersion interactions empirically or semiempirically at different levels of complexity. In this group are the PBE-D2, B97D, PBE-TS, PBE-XDM, and PBE+DCACP methods. The interaction energy curves of the second group are plotted in Fig. 4. The third group is composed by DFT-based methods that include dispersion interactions through non-local correlation functionals that do not depend on external parameters: the family of vdW-DF methods (vdW-DF, optB88-vdW, optB86b-vdW, vdW-DF-cx, and vdW-DF-C09), the family of vdW-DF2 methods (vdW-DF2, vdW-DF2-C09, and rev-vdWDF2), and the RVV10 method. Their corresponding energy curves are plotted in Fig. 5. In Subsections III A-III D, we compare the interaction energy curves of the methods studied with the $\operatorname{CCSD}(\mathrm{T})$ interaction energy curve of $\mathrm{H}_{2}$ on benzene.

\section{A. MP2 and DFT methods that do not include dispersion interactions vs $\operatorname{CCSD}(\mathrm{T})$}

First, we compare the MP2, VWN, PW91, and PBE results. The VWN, PW91, and PBE functionals do not include dispersion interactions. The VWN functional overestimates the $\mathrm{H}_{2}$-benzene interaction, resulting in a larger binding energy

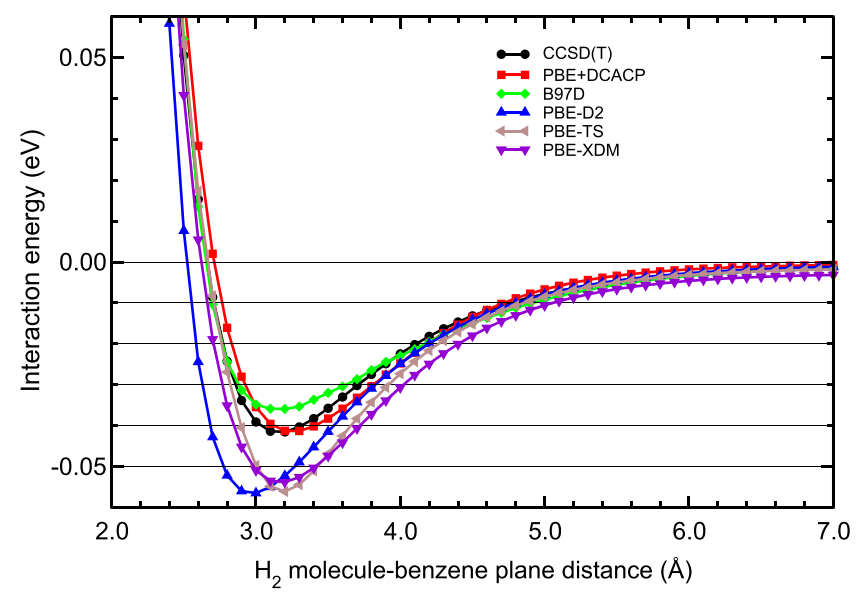

FIG. 4. Interaction energy between $\mathrm{H}_{2}$ and benzene as a function of the $\mathrm{H}_{2}$ benzene plane distance, obtained in CCSD(T) and DFT-D (PBE+DCACP, B97D, PBE-D2, PBE-TS, PBE-XDM) calculations.
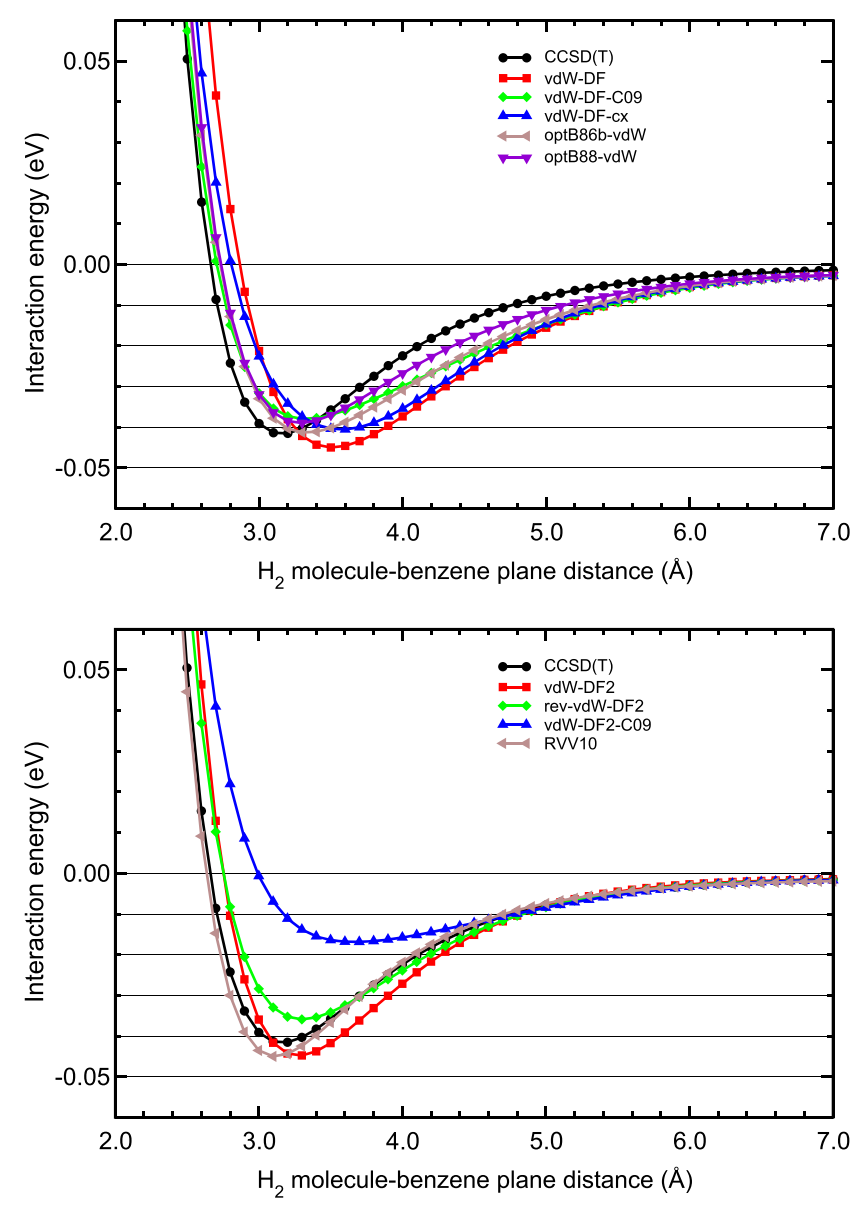

FIG. 5. Interaction energy between $\mathrm{H}_{2}$ and benzene as a function of the $\mathrm{H}_{2}$ benzene plane distance, obtained in $\operatorname{CCSD}(\mathrm{T})$ calculations and using the nonlocal functionals: The vdW-DF family, the vdW-DF2 family, and RVV10.

and a shorter equilibrium distance compare to the benchmark. The VWN binding energy is $-0.0996 \mathrm{eV}, 2.4$ times larger than the $\operatorname{CCSD}(\mathrm{T})$ binding energy. The VWN equilibrium distance is $2.8 \AA$, while the CCSD(T) equilibrium distance is $3.2 \AA$. The GGA functionals, PBE and PW91, underestimate the $\mathrm{H}_{2}$-benzene interaction, resulting in smaller binding energies and larger equilibrium distances. These two functionals yield the binding energies of -0.0204 and $-0.0290 \mathrm{eV}$, respectively, and a common equilibrium distance of $3.4 \AA$. Those features of the VWN, PW91, and PBE functionals are general and well known for other systems. The quantity $\mathrm{RMSE}_{m}$ measures all the differences between the curves. The values of RMSE ${ }_{m}$ of VWN, PW91, and PBE are very large, 0.0426, 0.0151 , and $0.0227 \mathrm{eV}$, respectively, indicating a large dissimilarity between these curves and the CCSD(T) curve. MP2 yields a binding energy of $-0.0487 \mathrm{eV}$, slightly larger than the $\operatorname{CCSD}(\mathrm{T})$ binding energy, an equilibrium distance of $3.1 \AA$, and a value of $\mathrm{RMSE}_{m}$ equal to $0.0074 \mathrm{eV}$, much smaller than the values of $\mathrm{RMSE}_{m}$ of the VWN, PW91, and PBE methods.

\section{B. DFT methods that include dispersion interactions empirically or semiempirically vs $\operatorname{CCSD}(\mathrm{T})$}

Second, we analyze the empirical and semiempirical DFT methods (PBE+DCACP, B97D, PBE-D2, PBE-TS, and PBE$\mathrm{XDM})$. The introduction of dispersion in the empirical and 
semiempirical DFT methods has a clear effect on improving the interaction energy curve between $\mathrm{H}_{2}$ and benzene, yielding better binding energies and equilibrium distances than the LDA and GGA methods. The binding energies of the DFT-D methods (B97D, PBE-D2, PBE-TS, and PBE$\mathrm{XDM}$ ) are within $11 \%-36 \%$ of the $\mathrm{CCSD}(\mathrm{T})$ binding energy. These results are consistent with several previous results on van der Waals clusters ${ }^{41,44,57,89-93}$ showing that DFT-D methods deliver binding energies within $10 \%-30 \%$ of those of the benchmark methods (CCSD(T) and MP2).

If we compare the interaction energy curves of the DFT$\mathrm{D}$ methods with the $\mathrm{CCSD}(\mathrm{T})$ interaction energy curve (see Fig. 4 and Table II), we find that B97D yields an interaction energy curve close to the $\operatorname{CCSD}(\mathrm{T})$ energy curve, with a small value of $\mathrm{RMSE}_{m}, 0.0035 \mathrm{eV}$, while the PBE-D2, PBETS, and PBE-XDM interaction energy curves are far from the $\operatorname{CCSD}(\mathrm{T})$ curve, with larger values of $\mathrm{RMSE}_{m}: 0.0132$, 0.0100 , and $0.0111 \mathrm{eV}$, respectively. The large dissimilarity between the interaction energy curves of PBE-D2, PBE-TS, and PBE-XDM, and the CCSD(T) curve, can be better noticed in the large values of their $\mathrm{RMSPE}_{m}: 44 \%, 28 \%$, and $34 \%$, respectively.

The DFT+DCACP method includes dispersion corrections through the dispersion corrected atom-centered pseudopotentials, DCACPs. The effect of those corrections can be obtained by making a comparison between PBE and $\mathrm{PBE}+\mathrm{DCACP}$ calculations of the interaction energy curve of $\mathrm{H}_{2}$ with benzene in Figs. 3 and 4. The difference in the binding energy, between PBE and PBE+DCACP, is about $0.02 \mathrm{eV}$, and the difference in the equilibrium distance is $0.1 \AA$. On the other hand, the PBE+DCACP binding energy is very close to the $\operatorname{CCSD}(\mathrm{T})$ binding energy: The difference in the binding energy is very small, $2 \times 10^{-4} \mathrm{eV}$. Even more important than the binding energy is the fact that the PBE+DCACP and the CCSD(T) interaction energy curves are in very good agreement, as can be seen in Fig. 4 and also in the small value of $\mathrm{RMSE}_{m}$ of the PBE+DCACP method, $0.0036 \mathrm{eV}$ (see Table II).

\section{DFT methods that include dispersion interactions through non-local functionals vs $\operatorname{CCSD}(\mathrm{T})$}

Third, the non-local functionals are analyzed: vdWDF, vdW-DF-C09, vdW-DF-cx, optB86b-vdW, optB88-vdW, vdW-DF2, rev-vdW-DF2, vdW-DF2-C09, and RVV10. The functionals of the vdW-DF and vdW-DF2 families, except the vdW-DF2-C09 functional, yield binding energies very close to the $\operatorname{CCSD}(\mathrm{T})$ binding energy: between $0 \%$ and $10 \%$ above or below the CCSD(T) binding energy (see Table II). However, the equilibrium $\mathrm{H}_{2}$-benzene plane distance of some of these functionals is far from the $\operatorname{CCSD}(\mathrm{T})$ equilibrium $\mathrm{H}_{2}$-benzene plane distance. On the other hand, RVV10 yields an equilibrium distance of $3.1 \AA$ and a binding energy of $-0.0450 \mathrm{eV}$, very close to the $\operatorname{CSD}(\mathrm{T})$ equilibrium distance and binding energy, $3.2 \AA$ and $-0.0415 \mathrm{eV}$, respectively.

The interaction energy curves obtained with the non-local functionals are plotted in Fig. 5. The binding energies of the functionals of the vdW-DF family are very close to the CCSD(T) binding energy. Their interaction energy curves are similar to the $\operatorname{CCSD}(\mathrm{T})$ interaction energy curve in the region around the minimum. However, they are very different from the CCSD(T) interaction energy curve in the tail region, as can be seen in Fig. 5 and especially in their values of $\mathrm{RMSPE}_{t}$ in Table II. The values of $\mathrm{RMSPE}_{t}$ of the vdW-DF family are very large: They range from $52 \%$ to $87 \%$, which means that in the tail region the interaction energy curves of this family are very different from the $\operatorname{CCSD}(\mathrm{T})$ curve.

As regards to the vdW-DF2 family, the interaction energy curves of the vdW-DF2 and rev-vdW-DF2 methods are close to the $\operatorname{CCSD}(\mathrm{T})$ interaction energy curve in both regions. Notice the low values of $\mathrm{RMSPE}_{t}$ of these two methods in Table II: $11 \%$ and $7 \%$, respectively. The interaction energy curve of vdW-DF2-C09 is very far from the $\operatorname{CCSD}(\mathrm{T})$ curve in the region around the minimum. Finally, the RVV10 curve is also close to the $\operatorname{CCSD}(\mathrm{T})$ curve in both regions, especially in the region around the minimum of the curve. Its values of $\mathrm{RMSE}_{m}$ and $\mathrm{RMSPE}_{m}$ are low: $0.0029 \mathrm{eV}$ and 9\%, respectively.

\section{DFT methods that best compare with CCSD(T) results of $\mathrm{H}_{2}$ on benzene}

From the plots of the interaction energy curves in Figs. 4 and 5 and the values of $\mathrm{RMSE}_{m}, \mathrm{RMSPE}_{m}, \mathrm{RMSE}_{t}$, and $\mathrm{RMSPE}_{t}$ in Table II, it can be concluded that, among the DFT methods studied, the RVV10, B97D, and PBE+DCACP interaction energy curves of $\mathrm{H}_{2}$ on benzene are the most similar to the $\operatorname{CCSD}(\mathrm{T})$ interaction energy curve. The agreement between the RVV10, B97D, and PBE+DCACP curves, and the $\operatorname{CCSD}(\mathrm{T})$ curve is remarkable, considering that the values of the interaction energy are very low. The values of $\mathrm{RMSE}_{m}$ of the RVV10, B97D, and PBE+DCACP methods are small: $0.0029,0.0035$, and $0.0036 \mathrm{eV}$, respectively. The B97D and


FIG. 6. The nine configurations of the $\mathrm{H}_{2}$ molecule on top of the graphene surface. Three sites: on carbon Atom, A, on carbon-carbon Bond, B, and on the center of the Hexagon, H. For each site, three different orientations of the molecular axis are explored. 
PBE+DCACP methods were built by fitting the parameters of these methods to data sets of high level ab initio calculations of weakly bonded systems ${ }^{41,45}$ that do not include $\mathrm{H}_{2}$-benzene. According to the present results, B97D and PBE+DCACP also yield results very similar to the $\operatorname{CCSD}(\mathrm{T})$ results for $\mathrm{H}_{2}$ on benzene.

\section{CALCULATIONS OF $\mathrm{H}_{2}$ PHYSISORBED ON GRAPHENE: DFT-BASED METHODS VS EXPERIMENTAL DATA}

Calculations of $\mathrm{H}_{2}$ physisorbed on graphene, on different sites and with different orientations of the molecular axis of $\mathrm{H}_{2}$ with respect to the graphene plane, have been carried out. PBE and different DFT-based methods that include dispersion interactions have been used. The molecule was placed on top of the three different sites of graphene: the center of a hexagon, $\mathrm{H}$, the center of a $\mathrm{C}-\mathrm{C}$ bond, $\mathrm{B}$, and a carbon atom, A. The three main orientations of the molecular axis of the $\mathrm{H}_{2}$ molecule have been studied: the axis perpendicular to the graphene plane, $\perp$, the axis parallel to the graphene plane and parallel to two $\mathrm{C}-\mathrm{C}$ bonds of the hexagon, \|\| , and the axis parallel to the graphene plane and perpendicular to two $\mathrm{C}-\mathrm{C}$ bonds of the hexagon, $\| \perp$. The combination of site and orientation is a configuration. We have studied the nine configurations of $\mathrm{H}_{2}$ on graphene plotted in Fig. 6. The following notation has been used for the configurations: The letter indicates the site where the molecule was placed and the set of

TABLE III. Equilibrium $\mathrm{H}_{2}$-graphene plane distances, $d_{e}$, in $\AA$, and binding energies, $E_{b}$, in $\mathrm{eV}$, for $\mathrm{H}_{2}$ on different sites on graphene and with different orientations of the molecular axis, obtained with the PBE, PBE+DCACP, PBE-D2, PBE-TS, PBE-XDM, methods, and the family of vdW-DF methods.

\begin{tabular}{|c|c|c|c|c|c|c|c|c|c|c|}
\hline \multirow[b]{2}{*}{ Configuration } & \multicolumn{2}{|c|}{ PBE } & \multicolumn{2}{|c|}{ PBE+DCACP } & \multicolumn{2}{|c|}{ PBE-D2 } & \multicolumn{2}{|c|}{ PBE-TS } & \multicolumn{2}{|c|}{ PBE-XDM } \\
\hline & $d_{e}$ & $E_{b}$ & $d_{e}$ & $E_{b}$ & $d_{e}$ & $E_{b}$ & $d_{e}$ & $E_{b}$ & $d_{e}$ & $E_{b}$ \\
\hline $\mathrm{A}_{\|\| \|}$ & 3.6 & -0.0093 & 3.3 & -0.0396 & 3.1 & -0.0489 & 3.1 & -0.0624 & 3.2 & -0.0486 \\
\hline $\mathrm{A}_{\| \perp}$ & 3.6 & -0.0093 & 3.3 & -0.0399 & 3.1 & -0.0489 & 3.1 & -0.0624 & 3.2 & -0.0486 \\
\hline $\mathrm{A}_{\perp}$ & 3.6 & -0.0111 & 3.3 & -0.0428 & 3.1 & -0.0577 & 3.2 & -0.0633 & 3.2 & -0.0513 \\
\hline $\mathrm{B}_{\|\| \|}$ & 3.6 & -0.0095 & 3.2 & -0.0405 & 3.0 & -0.0503 & 3.1 & -0.0637 & 3.2 & -0.0496 \\
\hline $\mathrm{B}_{\| \perp}$ & 3.5 & -0.0111 & 3.3 & -0.0396 & 3.1 & -0.0491 & 3.1 & -0.0630 & 3.2 & -0.0489 \\
\hline $\mathrm{B}_{\perp}$ & 3.5 & -0.0111 & 3.3 & -0.0431 & 3.1 & -0.0582 & 3.2 & -0.0634 & 3.2 & -0.0515 \\
\hline $\mathrm{H}_{\|\|}$ & 3.5 & -0.0110 & 3.1 & -0.0478 & 2.9 & -0.0608 & 2.9 & -0.0750 & 3.0 & -0.0567 \\
\hline $\mathrm{H}_{\| \perp}$ & 3.5 & -0.0110 & 3.1 & -0.0473 & 2.9 & -0.0608 & 2.9 & -0.0750 & 3.0 & -0.0567 \\
\hline \multirow[t]{2}{*}{$\mathrm{H}_{\perp}$} & 3.5 & -0.0116 & 3.2 & -0.0459 & 2.9 & -0.0641 & 3.1 & -0.0688 & 3.1 & -0.0543 \\
\hline & \multicolumn{2}{|c|}{ vdW-DF } & \multicolumn{2}{|c|}{ vdW-DF-C09 } & \multicolumn{2}{|c|}{ vdW-DF-cx } & \multicolumn{2}{|c|}{ optB86b-vdW } & \multicolumn{2}{|c|}{ optB88-vdW } \\
\hline Configuration & $d_{e}$ & $E_{b}$ & $d_{e}$ & $E_{b}$ & $d_{e}$ & $E_{b}$ & $d_{e}$ & $E_{b}$ & $d_{e}$ & $E_{b}$ \\
\hline$A_{\|\|}$ & 3.3 & -0.0761 & 3.2 & -0.0683 & 3.3 & -0.0708 & 3.2 & -0.0719 & 3.2 & -0.0686 \\
\hline $\mathrm{A}_{\| \perp}$ & 3.3 & -0.0761 & 3.2 & -0.0683 & 3.3 & -0.0708 & 3.2 & -0.0719 & 3.2 & -0.0686 \\
\hline $\mathrm{A}_{\perp}$ & 3.4 & -0.0748 & 3.2 & -0.0691 & 3.4 & -0.0693 & 3.2 & -0.0721 & 3.2 & -0.0698 \\
\hline $\mathrm{B}_{\|\| \|}$ & 3.3 & -0.0769 & 3.2 & -0.0692 & 3.3 & -0.0714 & 3.2 & -0.0728 & 3.2 & -0.0696 \\
\hline $\mathrm{B}_{\| \perp}$ & 3.3 & -0.0762 & 3.2 & -0.0684 & 3.3 & -0.0709 & 3.2 & -0.0720 & 3.2 & -0.0687 \\
\hline $\mathrm{B}_{\perp}$ & 3.4 & -0.0748 & 3.2 & -0.0693 & 3.4 & -0.0694 & 3.2 & -0.0724 & 3.2 & -0.0700 \\
\hline $\mathrm{H}_{\|\|}$ & 3.2 & -0.0798 & 3.1 & -0.0744 & 3.3 & -0.0744 & 3.1 & -0.0780 & 3.1 & -0.0752 \\
\hline $\mathrm{H}_{\| \perp}$ & 3.2 & -0.0798 & 3.1 & -0.0744 & 3.3 & -0.0744 & 3.1 & -0.0780 & 3.1 & -0.0752 \\
\hline $\mathrm{H}_{\perp}$ & 3.3 & -0.0754 & 3.1 & -0.0711 & 3.4 & -0.0703 & 3.2 & -0.0742 & 3.1 & -0.0716 \\
\hline
\end{tabular}

TABLE IV. Equilibrium $\mathrm{H}_{2}$-graphene plane distances, $d_{e}$, in $\AA$, and binding energies, $E_{b}$, in $\mathrm{eV}$, for $\mathrm{H}_{2}$ on different sites on graphene and with different orientations of the molecular axis, obtained with the family of vdW-DF2 methods and the RVV10 method.

\begin{tabular}{|c|c|c|c|c|c|c|c|c|}
\hline \multirow[b]{2}{*}{ Configuration } & \multicolumn{2}{|c|}{ vdW-DF2 } & \multicolumn{2}{|c|}{ rev-vdW-DF2 } & \multicolumn{2}{|c|}{ vdW-DF2-C09 } & \multicolumn{2}{|c|}{ RVV10 } \\
\hline & $d_{e}$ & $E_{b}$ & $d_{e}$ & $E_{b}$ & $d_{e}$ & $E_{b}$ & $d_{e}$ & $E_{b}$ \\
\hline $\mathrm{A}_{\|\|}$ & 3.2 & -0.0611 & 3.2 & -0.0511 & 3.4 & -0.0298 & 3.1 & -0.0532 \\
\hline $\mathrm{A}_{\| \perp}$ & 3.2 & -0.0611 & 3.3 & -0.0525 & 3.4 & -0.0298 & 3.1 & -0.0532 \\
\hline $\mathrm{A}_{\perp}$ & 3.2 & -0.0633 & 3.2 & -0.0527 & 3.4 & -0.0306 & 3.1 & -0.0577 \\
\hline $\mathrm{B}_{\|\|}$ & 3.2 & -0.0623 & 3.2 & -0.0521 & 3.4 & -0.0303 & 3.1 & -0.0546 \\
\hline $\mathrm{B}_{\| \perp}$ & 3.2 & -0.0611 & 3.2 & -0.0511 & 3.4 & -0.0298 & 3.1 & -0.0534 \\
\hline $\mathrm{B}_{\perp}$ & 3.2 & -0.0634 & 3.2 & -0.0529 & 3.4 & -0.0306 & 3.1 & -0.0580 \\
\hline $\mathrm{H}_{\|\|}$ & 3.1 & -0.0681 & 3.1 & -0.0575 & 3.3 & -0.0326 & 3.0 & -0.0627 \\
\hline $\mathrm{H}_{\| \perp}$ & 3.1 & -0.0681 & 3.1 & -0.0575 & 3.3 & -0.0326 & 3.0 & -0.0627 \\
\hline $\mathrm{H}_{\perp}$ & 3.2 & -0.0646 & 3.2 & -0.0548 & 3.4 & -0.0311 & 3.1 & -0.0611 \\
\hline
\end{tabular}


symbols after the letter denotes the orientation of the molecular axis.

The binding energies of $\mathrm{H}_{2}$ on graphene and the equilibrium $\mathrm{H}_{2}$-graphene surface distances obtained with different DFT methods for the nine configurations of $\mathrm{H}_{2}$ on graphene are shown in Tables III and IV. The site with the lowest binding energies for all the DFT methods studied in Tables III and IV is the center of the hexagon, site $\mathrm{H}$. The binding energies of $\mathrm{H}_{2}$ on the $\mathrm{C}-\mathrm{C}$ bond, site $\mathrm{B}$, and on the carbon atom, site $\mathrm{A}$, are less negative. The lowest binding energy in Tables III and IV, for most of the DFT methods studied, corresponds to the $\mathrm{H}_{\|\|}$


FIG. 7. Interaction energy between $\mathrm{H}_{2}$ and graphene as a function of the $\mathrm{H}_{2}$ graphene plane distance, obtained with PBE and with different DFT-based methods that include dispersion interactions. $\mathrm{H}_{2}$ is in the configuration $\mathrm{H}_{\|\| \|}$. configuration. Nevertheless, the other two orientations of the molecule on site $\mathrm{H}$ have binding energies close to the $\mathrm{H}_{\|||}$configuration. Since the $\mathrm{H}_{\|||}$configuration has the lowest binding energies, the interaction energy curves have been calculated only for the $\mathrm{H}_{\|\|}$configuration and are plotted in Fig. 7.

The theoretical results should be compared with experiments. Mattera et al. ${ }^{64}$ measured the energy of the lowest level of $\mathrm{H}_{2}$ physisorbed on graphite. They reported a value of $-0.04161 \mathrm{eV}$, with an experimental accuracy of $0.00025 \mathrm{eV}$. Costanzo et al. ${ }^{52}$ used a model ${ }^{94}$ to discount the effect of the other graphene layers of graphite, in order to obtain the experimental energy of the lowest level of $\mathrm{H}_{2}$ physisorbed on graphene. They found that the energy should be corrected by $6 \times 10^{-3} \mathrm{eV}$. The adsorption energy obtained by Mattera et al. ${ }^{64}$ on graphite, $-0.0416 \mathrm{eV}$, turned out to be $-0.0476 \mathrm{eV}$ on graphene, according to that model. This is the experimental value of the adsorption energy of the lowest level of $\mathrm{H}_{2}$ physisorbed on graphene, $\mathrm{E}_{a d s, \exp }$.

To obtain the adsorption energy of the lowest level of $\mathrm{H}_{2}$ physisorbed on graphene, $\mathrm{E}_{a d s}$, we have to calculate the zero point energy of the interaction energy curve, $\mathrm{E}_{z p}$, and subtract it from the binding energy, i.e., $\mathrm{E}_{a d s}=\mathrm{E}_{b}-\mathrm{E}_{z p}$. We have calculated the zero point energy $\mathrm{E}_{z p}$ of the interaction energy curve obtained with every DFT-based method, for the $\mathrm{H}_{\|\|}$ configuration and later, the adsorption energy of the lowest level of $\mathrm{H}_{2}$ physisorbed on graphene, $\mathrm{E}_{a d s}=\mathrm{E}_{b}-\mathrm{E}_{z p}$.

The results of the calculations of $\mathrm{H}_{2}$ on graphene for the $\mathrm{H}_{\|\|}$configuration are shown in Table V. To calculate accurately the zero point energy, we have done more calculations for $\mathrm{H}_{2}$-graphene distances $d$ around the minimum, with a step of $0.01 \AA$. Therefore the results in Table $\mathrm{V}$ are slightly different from the results of the configuration $\mathrm{H}_{\|||}$in Tables III and IV.

The theoretical value of the adsorption energy of the lowest level of $\mathrm{H}_{2}$ on graphene, $\mathrm{E}_{a d s}$, is also compared with the experimental value, $\mathrm{E}_{a d s, \exp }$, in that table. The adsorption energy of the lowest level obtained with the PBE-D2,

TABLE V. Equilibrium $\mathrm{H}_{2}$-graphene plane distances, $d_{e}$, in $\AA$, binding energies, $E_{b}$, in eV, and adsorption energies of the lowest level, $E_{a d s}$, for $\mathrm{H}_{2}$ on configuration $\mathrm{H}_{\|\| \|}$, obtained with different DFT methods. Error $=E_{a d s}$ $-E_{a d s, \text { exp }}$ is in $\mathrm{eV}$ and Relative error $=100$ Error $/\left|E_{\text {ads, exp }}\right|$ is in $\%$.

\begin{tabular}{lccccc}
\hline \hline Method & $d_{e}$ & $E_{b}$ & $E_{a d s}$ & Error & Relative error \\
\hline PBE & 3.46 & -0.0110 & -0.0062 & 0.0414 & 87.0 \\
PBE+DCACP & 3.06 & -0.0478 & -0.0382 & 0.0094 & 19.7 \\
PBE-D2 & 2.85 & -0.0610 & -0.0507 & -0.0031 & -6.5 \\
PBE-TS & 2.94 & -0.0754 & -0.0610 & -0.0134 & -28.2 \\
PBE-XDM & 3.05 & -0.0568 & -0.0470 & 0.0006 & 1.3 \\
\hline vdW-DF & 3.24 & -0.0800 & -0.0706 & -0.0230 & -48.3 \\
vdW-DF-C09 & 3.06 & -0.0745 & -0.0657 & -0.0181 & -38.0 \\
vdW-DF-cx & 3.26 & -0.0745 & -0.0666 & -0.0190 & -39.9 \\
optB86b-vdW & 3.08 & -0.0780 & -0.0686 & -0.0210 & -44.1 \\
optB88-vdW & 3.06 & -0.0754 & -0.0656 & -0.0180 & -37.8 \\
\hline vdW-DF2 & 3.06 & -0.0682 & -0.0573 & -0.0097 & -20.4 \\
rev-vdW-DF2 & 3.07 & -0.0576 & -0.0481 & -0.0005 & -1.1 \\
vdW-DF2-C09 & 3.28 & -0.0326 & -0.0266 & 0.0210 & 44.1 \\
RVV10 & 2.96 & -0.0629 & -0.0520 & -0.0044 & -9.2 \\
\hline \hline
\end{tabular}


PBE-XDM, rev-vdW-DF2, and RVV10 methods is close to the experimental value: within $5 \times 10^{-3} \mathrm{eV}$ and $10 \%$ of the experimental value, $\mathrm{E}_{a d s, \exp }=-0.0476 \mathrm{eV}$. The adsorption energies obtained with the other DFT methods are less accurate.

\section{SUMMARY AND CONCLUSIONS}

The goal of this work has been to search for DFT-based methods that reproduce accurately the physisorption interaction between $\mathrm{H}_{2}$ and benzene and also between $\mathrm{H}_{2}$ and graphene, in particular, DFT-based methods that reproduce the CCSD(T) interaction energy curve of $\mathrm{H}_{2}$ on benzene and the experimental data of the physisorption of $\mathrm{H}_{2}$ on graphene. To reach this goal, calculations of the interaction energy curves of $\mathrm{H}_{2}$ on benzene using the $\operatorname{CCSD}(\mathrm{T}), \mathrm{MP} 2, \mathrm{PBE}$, VWN, PW91, PBE+DCACP, B97D, PBE-D2, PBE-TS, PBEXDM, vdW-DF, vdW-DF-C09, vdW-DF-cx, ptB86b-vdW, optB88-vdW, vdW-DF2, rev-vdW-DF2, vdW-DF2-C09, and RVV10 methods, and calculations of the interaction energy curves of $\mathrm{H}_{2}$ on graphene using the PBE, PBE+DCACP, PBE-D2, PBE-TS, PBE-XDM, vdW-DF, vdW-DF-C09, vdWDF-cx, optB86b-vdW, optB88-vdW, vdW-DF2, rev-vdWDF2, vdW-DF2-C09, and RVV10 methods, have been carried out.

The most relevant conclusions are as follows: (a) The B97D, RVV10, and PBE+DCACP methods yield interaction energy curves of $\mathrm{H}_{2}$-benzene that reproduce rather well the $\operatorname{CCSD}(\mathrm{T})$ interaction energy curve: within $4 \times 10^{-3} \mathrm{eV}$ and $13 \%$, on average, of the $\operatorname{CCSD}(\mathrm{T})$ interaction energy curve in the region around the minimum (see Figs. 4 and 5, and Table II). Therefore, these three DFT-based methods provide a good description of the physisorption of $\mathrm{H}_{2}$ on benzene, with a good compromise between accuracy and computer cost. (b) The rev-vdW-DF2, PBE-XDM, PBE-D2, and RVV10 methods yield adsorption energies of the lowest level of $\mathrm{H}_{2}$ on graphene very close to the experimental result: within $5 \times 10^{-3}$ $\mathrm{eV}$ and $10 \%$ of the experimental value, $-0.0476 \mathrm{eV}$. Hence, these DFT methods can be used to obtain a good description of the physisorption of $\mathrm{H}_{2}$ on graphene, with a good compromise between accuracy and computer cost. Among all the DFT methods studied in the present work, RVV10 is the method giving the most accurate physisorption results in both, benzene and graphene. These conclusions are of particular interest for the investigation of hydrogen storage on porous carbon materials.

\section{ACKNOWLEDGMENTS}

This work was supported by MINECO of Spain (Grant No. MAT2014-54378-R), Junta de Castilla y León (Grant No. VA050U14), and the University of Valladolid. We acknowledge the facilities provided by Centro de Proceso de DatosParque Científico of the University of Valladolid.

\footnotetext{
${ }^{1}$ P. Bernard and R. Chahine, Langmuir 17, 1950 (2001).

${ }^{2}$ Y. Gogotsi, A. Nikitin, H. Ye, W. Zhou, J. E. Fischer, B. Yi, H. C. Foley, and M. W. Barsoum, Nat. Mater. 2, 591 (2003).

${ }^{3}$ S. Challet, P. Azaïs, R. J.-M. Pellenq, O. Isnard, J.-L. Soubeyroux, and L. Duclaux, J. Phys. Chem. Solids 65, 541 (2004).

${ }^{4}$ Y. Gogotsi, R. K. Dash, G. Yushin, T. Yildirim, G. Laudisio, and J. E. Fischer, J. Am. Chem. Soc. 127, 16006 (2005).
}

${ }^{5}$ G. Yushin, R. Dash, J. Jagiello, J. E. Fischer, and Y. Gogotsi, Adv. Funct. Mater. 16, 2288 (2006).

${ }^{6}$ M. Jordá-Beneyto, F. Suárez-García, D. Lozano-Castelló, D. CazorlaAmorós, and A. Linares-Solano, Carbon 45, 293 (2007).

${ }^{7}$ M. Jordá-Beneyto, D. Lozano-Castelló, F. Suárez-García, D. CazorlaAmorós, and A. Linares-Solano, Microporous Mesoporous Mater. 112, 235 (2008).

${ }^{8}$ Y. Gogotsi, C. Portet, S. Osswald, J. M. Simmons, T. Yildirim, G. Laudisio, and J. E. Fischer, Int. J. Hydrogen Energy 34, 6314 (2009).

${ }^{9}$ F. Suárez-García, E. Vilaplana-Ortego, M. Kunowsky, M. Kimura, A. Oya, and A. Linares-Solano, Int. J. Hydrogen Energy 34, 9141 (2009).

${ }^{10}$ I. Cabria, M. J. López, and J. A. Alonso, in Handbook of Nanophysics: Functional Nanomaterials, edited by K. D. Sattler (CRC Press, Boca Raton, 2010), Chap. 41, pp. 41-1-41-16.

${ }^{11}$ M. Kunowsky, J. P. Marco-Lozar, A. Oya, and A. Linares-Solano, Carbon 50, 1407 (2012)

${ }^{12}$ G. G. I. Wróbel-Iwaniec and N. Díez, Int. J. Hydrogen Energy 40, 5788 (2015).

${ }^{13}$ G. Sethia and A. Sayari, Carbon 99, 289 (2016).

${ }^{14}$ M. Rzepka, P. Lamp, and M. A. de la Casa-Lillo, J. Phys. Chem. B 102, 10894 (1998)

${ }^{15}$ Q. Wang and J. K. Johnson, Mol. Phys. 95, 299 (1998).

${ }^{16}$ Q. Wang and J. K. Johnson, J. Chem. Phys. 110, 577 (1999).

${ }^{17}$ S. Patchkovskii, J. S. Tse, S. N. Yurchenko, L. Zhechkov, T. Heine, and G. Seifert, Proc. Natl. Acad. Sci. U. S. A. 102, 10439 (2005).

${ }^{18}$ I. Cabria, M. J. López, and J. A. Alonso, Carbon 45, 2649 (2007).

${ }^{19}$ G. Mpourmpakis and G. E. Froudakis, J. Nanosci. Nanotechnol. 8, 3091 (2008).

${ }^{20}$ I. Cabria, M. J. López, and J. A. Alonso, J. Chem. Phys. 128, 144704 (2008).

${ }^{21}$ I. Cabria, M. J. López, and J. A. Alonso, Phys. Rev. B 78, 075415 (2008).

${ }^{22}$ Y. Ihm, V. R. Cooper, L. Peng, and J. R. Morris, J. Phys.: Condens. Matter 24, 424205 (2012).

${ }^{23}$ J. A. Alonso, I. Cabria, and M. J. López, J. Mater. Res. 28, 589 (2013).

${ }^{24}$ J. Čížek, J. Chem. Phys. 45, 4256 (1966).

${ }^{25}$ C. Møller and M. S. Plesset, Phys. Rev. 46, 618 (1934).

${ }^{26}$ K. E. Riley, M. Pitoňák, P. Jurečka, and P. Hobza, Chem. Rev. 110, 5023 (2010).

${ }^{27}$ K. E. Riley and P. Hobza, Wiley Interdiscip. Rev.: Comput. Mol. Sci. 1, 3 (2011).

${ }^{28}$ P. Hobza, Acc. Chem. Res. 45, 663 (2012).

${ }^{29}$ J. Klimeš and A. Michaelides, J. Chem. Phys. 137, 120901 (2012).

${ }^{30}$ M. Dion, H. Rydberg, E. Schröder, D. C. Langreth, and B. I. Lundqvist, Phys. Rev. Lett. 92, 246401 (2004).

${ }^{31}$ P. Hyldgaard, K. Berland, and E. Schröder, Phys. Rev. B 90, 075148 (2014).

${ }^{32}$ J. Klimeš, D. R. Bowler, and A. Michaelides, J. Phys.: Condens. Matter 22, $022201(2010)$.

${ }^{33}$ J. Klimeš, D. R. Bowler, and A. Michaelides, Phys. Rev. B 83, 195131 (2011).

${ }^{34}$ K. Berland and P. Hyldgaard, Phys. Rev. B 89, 035412 (2014).

${ }^{35}$ V. R. Cooper, Phys. Rev. B 81, 161104(R) (2010).

${ }^{36}$ K. Lee, E. D. Murray, L. Kong, B. I. Lundqvist, and D. C. Langreth, Phys. Rev. B 82, 081101(R) (2010).

${ }^{37}$ I. Hamada and M. Otani, Phys. Rev. B 82, 153412 (2010).

${ }^{38}$ I. Hamada, Phys. Rev. B 89, 121103(R) (2014).

${ }^{39}$ O. A. Vydrov and T. van Voorhis, J. Chem. Phys. 133, 244103 (2010).

${ }^{40}$ R. Sabatini, T. Gorni, and S. de Gironcoli, Phys. Rev. B 87, 041108(R) (2013).

${ }^{41}$ S. Grimme, J. Comput. Chem. 27, 1787 (2006).

${ }^{42}$ O. A. von Lilienfeld, I. Tavernelli, U. Rothlisberger, and D. Sebastiani, Phys. Rev. Lett. 93, 153004 (2004).

${ }^{43}$ O. A. von Lilienfeld, I. Tavernelli, U. Rothlisberger, and D. Sebastiani, Phys. Rev. B 71, 195119 (2005).

${ }^{44}$ E. Tapavicza, I. C. Lin, O. A. von Lilienfeld, I. Tavernelli, M. D. CoutinhoNeto, and U. Rothlisberger, J. Chem. Theory Comput. 3, 1673 (2007).

${ }^{45}$ I. Lin, M. D. Coutinho-Neto, C. Felsenheimer, O. A. von Lilienfeld, I. Tavernelli, and U. Rothlisberger, Phys. Rev. B 75, 205131 (2007).

${ }^{46}$ See http://lcbc.epfl.ch/page-71135-en.html for Library of DispersionCorrected Atom-Centered Potentials (accessed May 11, 2017).

${ }^{47}$ A. Tkatchenko and M. Scheffler, Phys. Rev. Lett. 102, 073005 (2009).

${ }^{48}$ A. D. Becke and E. R. Johnson, J. Chem. Phys. 127, 154108 (2007).

${ }^{49}$ A. O. de la Roza and E. R. Johnson, J. Chem. Phys. 136, 174109 (2012).

${ }^{50}$ P. L. Silvestrelli, Phys. Rev. Lett. 100, 053002 (2008). 
${ }^{51}$ P. L. Silvestrelli, J. Phys. Chem. A 113, 5224 (2009).

${ }^{52}$ F. Costanzo, P. L. Silvestrelli, and F. Ancilotto, J. Chem. Theory Comput. 8, 1288 (2012).

${ }^{53}$ A. Ambrosetti and P. L. Silvestrelli, Phys. Rev. B 85, 073101 (2012).

${ }^{54}$ P. L. Silvestrelli and A. Ambrosetti, J. Chem. Phys. 140, 124107 (2014).

${ }^{55}$ Y. Zhao and D. G. Truhlar, J. Chem. Theory Comput. 1, 415 (2005).

${ }^{56}$ Q. Hill and C.-K. Skylaris, Proc. R. Soc. A 465, 669 (2009).

${ }^{57}$ C. D. Sherrill, T. Takatani, and E. G. Hohenstein, J. Phys. Chem. A 113, 10146 (2009).

${ }^{58}$ Y. Liu and W. A. Goddard III, J. Phys. Chem. Lett. 1, 2550 (2010).

${ }^{59}$ K. S. Thanthiriwatte, E. G. Hohenstein, L. A. Burns, and C. D. Sherrill, J. Chem. Theory Comput. 7, 88 (2011).

${ }^{60}$ J. C. Flick, D. Kosenkov, E. G. Hohenstein, C. D. Sherrill, and L. V. Slipchenko, J. Chem. Theory Comput. 8, 2835 (2012).

${ }^{61}$ M. Doemer, I. Tavernelli, and U. Rothlisberger, J. Chem. Theory Comput. 9, 955 (2013).

${ }^{62}$ M. Callsen and I. Hamada, Phys. Rev. B 91, 195103 (2015).

${ }^{63}$ I. Cabria, M. J. López, and J. A. Alonso, Int. J. Hydrogen Energy 36, 10748 (2011).

${ }^{64}$ L. Mattera, R. Rosatelli, C. Salvo, F. Tommasini, U. Valbusa, and G. Vidali, Surf. Sci. 93, 515 (1980).

${ }^{65}$ J. P. Perdew, K. Burke, and M. Ernzerhof, Phys. Rev. Lett. 77, 3865 (1996).

${ }^{66}$ J. P. Perdew, K. Burke, and M. Ernzerhof, Phys. Rev. Lett. 78, 1396 (1997).

${ }^{67}$ S. H. Vosko, L. Wilk, and M. Nusair, Can. J. Phys. 58, 1200 (1980).

${ }^{68}$ J. P. Perdew and Y. Wang, Phys. Rev. B 45, 13244 (1992).

${ }^{69}$ A. D. Becke, J. Chem. Phys. 107, 8554 (1997).

${ }^{70}$ M. J. Frisch, G. W. Trucks, H. B. Schlegel, G. E. Scuseria, M. A. Robb, J. R. Cheeseman, G. Scalmani, V. Barone, B. Mennucci, G. A. Petersson et al., GaUSSIAN 09, Revision B.01, Gaussian, Inc., Wallingford, CT, 2009.

${ }^{71}$ L. Genovese, A. Neelov, S. Goedecker, T. Deutsch, S. A. Ghasemi, A. Willand, D. Caliste, O. Zilberberg, M. Rayson, A. Bergman et al., J. Chem. Phys. 129, 014109 (2008).

${ }^{72}$ T. Deutsch and L. Genovese, Collect. SFN 12, 33 (2011).

${ }^{73}$ See http://bigdft.org for BigDFT Code (accessed May 11, 2017).
${ }^{74}$ See http://www.quantum-espresso.org for Quantum Espresso Code (accessed May 11, 2017).

${ }^{75}$ P. Giannozzi, S. Baroni, N. Bonini, M. Calandra, R. Car, C. Cavazzoni, D. Ceresoli, G. L. Chiarotti, M. Cococcioni, I. Dabo et al., J. Phys.: Condens. Matter 21, 395502 (2009).

${ }^{76}$ T. H. Dunning, Jr., J. Chem. Phys. 90, 1007 (1989).

${ }^{77}$ R. A. Kendall, T. H. Dunning, Jr., and R. J. Harrison, J. Chem. Phys. 96, 6796 (1992).

${ }^{78}$ D. E. Woon and T. H. Dunning, Jr., J. Chem. Phys. 98, 1358 (1993).

${ }^{79}$ K. A. Peterson, D. E. Woon, and T. H. Dunning, Jr., J. Chem. Phys. 100, 7410 (1994)

${ }^{80}$ E. R. Davidson, Chem. Phys. Lett. 260, 514 (1996).

${ }^{81}$ S. F. Boys and F. Bernardi, Mol. Phys. 19, 553 (1970).

${ }^{82}$ T. Thonhauser, V. R. Cooper, S. Li, A. Puzder, P. Hyldgaard, and D. C. Langreth, Phys. Rev. B 76, 125112 (2007).

${ }^{83}$ G. Román-Pérez and J. M. Soler, Phys. Rev. Lett. 103, 096102 (2009).

${ }^{84}$ M. Troullier and J. L. Martins, Phys. Rev. B 43, 1993 (1991).

${ }^{85} \mathrm{G}$. Kresse and J. Joubert, Phys. Rev. B 59, 1758 (1999).

${ }^{86}$ J. S. Sims and S. A. Hagstrom, J. Chem. Phys. 124, 094101 (2006).

${ }^{87}$ J. Plíva, J. W. C. Johns, and L. Goodman, J. Mol. Spectrosc. 148, 427 (1991).

${ }^{88}$ R. W. G. Wyckoff, Crystal Structures (Interscience, New York, 1963), Vol. 1.

${ }^{89}$ P. Jurečka, J. Cerny, P. Hobza, and D. R. Salahub, J. Comput. Chem. 28, 555 (2007).

${ }^{90}$ M. Kabeláč, H. Valdés, E. C. Sherer, C. J. Cramer, and P. Hobza, Phys. Chem. Chem. Phys. 9, 5000 (2007).

${ }^{91}$ C. Morgado, J. McNamara, I. Hillier, N. Burton, and M. Vincent, J. Chem. Theory Comput. 3, 1656 (2007)

${ }^{92}$ K. E. Riley, J. Vondrášek, and P. Hobza, Phys. Chem. Chem. Phys. 9, 5555 (2007).

${ }^{93}$ A. Vázquez-Mayagoitia, C. D. Sherrill, E. Aprà, and B. G. Sumpter, J. Chem. Theory Comput. 6, 727 (2010).

${ }^{94}$ A. D. Crowell and J. S. Brown, Surf. Sci. 123, 296 (1982). 